\title{
Probing the Anomalous FCNC tq $\gamma$ Couplings at Large Hadron Electron Collider
}

\author{
I. Turk Cakir, ${ }^{1,2}$ A. Yilmaz, ${ }^{3}$ H. Denizli, ${ }^{4}$ A. Senol, ${ }^{4}$ H. Karadeniz, ${ }^{1}$ and O. Cakir ${ }^{5}$ \\ ${ }^{1}$ Department of Energy Systems Engineering, Giresun University, 28200 Giresun, Turkey \\ ${ }^{2}$ European Organization for Nuclear Research (CERN), Geneva, Switzerland \\ ${ }^{3}$ Department of Electric and Electronics Engineering, Giresun University, 28200 Giresun, Turkey \\ ${ }^{4}$ Department of Physics, Abant Izzet Baysal University, 14280 Bolu, Turkey \\ ${ }^{5}$ Department of Physics, Ankara University, Tandogan, 06100 Ankara, Turkey
}

Correspondence should be addressed to I. Turk Cakir; ilkay.turk.cakir@cern.ch

Received 6 May 2017; Accepted 14 August 2017; Published 14 September 2017

Academic Editor: Jinmian Li

Copyright (C) 2017 I. Turk Cakir et al. This is an open access article distributed under the Creative Commons Attribution License, which permits unrestricted use, distribution, and reproduction in any medium, provided the original work is properly cited. The publication of this article was funded by SCOAP $^{3}$.

We investigate the anomalous flavour changing neutral current (FCNC) interactions of top quark through the process $e^{-} p \rightarrow$ $e^{-} W^{ \pm} q+X$. We calculate the signal and background cross sections in electron-proton collisions at Large Hadron electron Collider $(\mathrm{LHeC})$ with a $7 \mathrm{TeV}$ proton beam from the $\mathrm{LHC}$ and a new $60 \mathrm{GeV}$ electron beam from energy recovery linac (ERL). We study the relevant background processes including one electron and three jets in the final state. The distributions of the invariant mass of two jets and an additional jet tagged as $b$-jet are used to account for signal and background events after the analysis cuts. We find upper bounds on anomalous FCNC couplings $\lambda$ of the order of $10^{-2}$ at $\mathrm{LHeC}$ for a luminosity projection of $500 \mathrm{fb}^{-1}$ together with the fast simulation of detector effects. As a matter of interest, we analyze the sensitivity to the couplings $\left(\lambda_{u}, \lambda_{c}\right)$ and find an enhanced sensitivity to $\lambda_{c}$ at the $\mathrm{LHeC}$ when compared to the results from the HERA.

\section{Introduction}

The top quark is the most massive of all observed elementary particles. The top quark interacts with the other quarks of Standard Model (SM) via gauge and Yukawa couplings. It interacts primarily by the strong interaction, but can only decay through the weak interaction. The top quark decays to a $W$ boson and either a bottom quark (most frequently), a strange quark, or a down quark (rarely). It is also a unique probe to search for the dynamics of electroweak symmetry breaking. Being the heaviest quark, effects of new physics on its couplings are expected to be larger than that for other fermions; hence it is expected that possible deviations with respect to predictions of the SM might be found with precise measurements of its couplings. It is known that the flavour changing neutral current (FCNC) transitions are absent at tree level and highly suppressed at loop level due to the Glashow-Iliopoulos-Maiani (GIM) mechanism [1]. The branching ratios for the top quark FCNC decays through the process $t \rightarrow q \gamma$ are of the order $10^{-14}-10^{-12}$ in the SM [2]. However, various scenarios beyond the standard model (BSM), such as two Higgs doublet model, supersymmetry, and technicolor, predict much larger rates at the order of $10^{-6}-10^{-5}[3,4]$. Therefore, an observation of the large FCNC induced couplings would indicate the existence of the BSM.

The experimental limits on the branching ratios of the rare top quark decays were established by the experiments at Tevatron and LHC. The CDF experiment set upper bound on branching ratio for top quark FCNC decay as $\mathrm{BR}(t \rightarrow q \gamma)<$ $3.2 \times 10^{-2}$ [5]. Presently, the most powerful upper limits on top quark FCNC branching ratios from different channels are $\mathrm{BR}(t \rightarrow u \gamma)<1.61 \times 10^{-4}$ and $\mathrm{BR}(t \rightarrow c \gamma)<1.82 \times 10^{-3}$ at 95\% confidence level (CL) given by the CMS experiment [6].

The direct single top quark production channels through the photon emission at the LHC have been studied to probe the top quark FCNC tq $\gamma$ interactions [7, 8]. Search for flavour changing neutral currents in the single top association with a 
photon at the LHC has been studied in [9]. In the same sign top quark production at the LHC the top FCNC couplings have been studied in [10]. The top FCNC couplings at future circular hadron electron colliders have been studied in [11].

The main physics goals of future high energy particle colliders are to examine the fundamental interactions within the SM and to search for possible effects of new physics beyond the SM. The electron-proton colliders are based on a ring type proton accelerator with an intersecting electron beam accelerator. Currently, a projected future ep collider, namely, Large Hadron electron Collider ( $\mathrm{LHeC}$ ), has been discussed [12-14]. It comprises a $60 \mathrm{GeV}$ electron beam that will collide with the $7 \mathrm{TeV}$ proton beam, having an integrated luminosity of $L_{\text {int }}=100 \mathrm{fb}^{-1}$ per year, and it is planned to collect $1 \mathrm{ab}^{-1}$ over the years. Recently, the new physics capability and potential of ep colliders through di-Higgs boson production have been studied in [15].

When we study anomalous top quark FCNC interactions at the future colliders, we take also into account bounds from measured low energy processes where the loops include top quarks. For example, the FCNC transition processes $b \rightarrow$ $s \gamma$ are considered to be the valuable probe of top quark anomalous couplings [16, 17]. The bounds [18] on the top quark real FCNC couplings are lower than the current direct limit but still accessible at the high luminosity run of LHC.

In this work, we study the physics potential of $\mathrm{LHeC}$ collider two parameters for probing top quark tq $\gamma$ FCNC couplings through single top production. We focus on two parameters analysis of the signal and background including Delphes detector simulation. We discuss the sensitivity and bounds for anomalous top quark couplings $\left(\lambda_{u}, \lambda_{c}\right)$ for different luminosity projections.

\section{Calculation Framework}

The top quark FCNC interactions would be a good test of new physics at present and future colliders. The new physics effects can be described by a set of higher order effective operators in a model independent framework. The anomalous FCNC interactions of top quark with up-type quarks $(u, c)$ and a photon can be described in a model independent effective Lagrangian

$$
\begin{aligned}
L_{\mathrm{eff}}= & \frac{g_{e}}{2 m_{t}} \bar{t} \sigma^{\mu \nu}\left(\lambda_{u}^{L} P_{L}+\lambda_{u}^{R} P_{R}\right) u A_{\mu \nu} \\
& +\frac{g_{e}}{2 m_{t}} \bar{t} \sigma^{\mu \nu}\left(\lambda_{c}^{L} P_{L}+\lambda_{c}^{R} P_{R}\right) c A_{\mu \nu}+\text { h.c. }
\end{aligned}
$$

where $g_{e}$ is the electromagnetic coupling constant, $\lambda_{q}^{L(R)}$ are the strength of anomalous FCNC couplings and the values of these vanish at the lowest order in the SM, and $P_{L(R)}$ denotes the left (right) handed projection operators. The photon field strength tensor is $A_{\mu \nu}$ and anomalous term is related to $\sigma^{\mu \nu}=$ $(i / 2)\left[\gamma^{\mu}, \gamma^{\nu}\right]$. The effective Lagrangian is used to calculate both production cross section and the decay widths for the $t \rightarrow q \gamma$ channels. At the ep colliders anomalous single top quark production can be achieved through the scattering of incoming electron beam with the quark current of the proton
TABLE 1: The signal cross section values (in $\mathrm{pb}$ ) for process $e^{-} p \rightarrow$ $\left(e^{-} t+e^{-} \bar{t}\right)+X$ at LHeC.

\begin{tabular}{lccc}
\hline LHeC & $\lambda_{c}=10^{-2}$ & $\lambda_{c}=10^{-3}$ & $\lambda_{c}=0$ \\
\hline$\lambda_{u}=10^{-2}$ & $9.468 \times 10^{-3}$ & $8.368 \times 10^{-3}$ & $8.357 \times 10^{-3}$ \\
$\lambda_{u}=10^{-3}$ & $1.188 \times 10^{-3}$ & $9.460 \times 10^{-5}$ & $8.365 \times 10^{-5}$ \\
$\lambda_{u}=0$ & $1.103 \times 10^{-3}$ & $1.104 \times 10^{-5}$ & 0 \\
\hline
\end{tabular}

via the production channel ( $t$-channel) as shown in diagrams, Figure 1. For the event generation and cross section calculations, we have used event generator MadGraph5_aMC@NLO [19] with built in parton distribution function NNPDF23 [20]. The effective interactions described by (1) have been implemented through FeynRules [21] and the model file is used within MadGraph5_aMC@NLO. This event generator provides the tools to perform the simulation of both signal and backgrounds within the same framework. In this study, an allowed range of values for the FCNC parameters $\lambda_{q}^{L(R)} \leq$ 0.05 is taken into account.

\section{Cross Section for the Signal}

The contributions from new interactions of the BSM physics can modify the production rate and properties of the top quark expected within the SM. The relative contribution of the left and right currents is determined by $\lambda_{q}^{L}$ and $\lambda_{q}^{R}$ as given in (1); no specific chirality is assumed for the FCNC interaction vertices $(t q \gamma)$; here we use a simplified scenario with $\lambda_{q}^{L}=\lambda_{q}^{R}=\lambda_{q}$ throughout the study. We study single top quark (or anti-top quark) production through the FCNC interactions at the LHeC. The signal cross sections for the processes $e^{-} p \rightarrow e^{-} t+X$ and $e^{-} p \rightarrow e^{-\bar{t}}+X$ are $\sigma(t)=$ $196.90 \mathrm{fb}$ and $\sigma(\bar{t})=40.21 \mathrm{fb}$ for equal coupling scenario $\lambda=\lambda_{u}=\lambda_{c}=0.05$ at the center of mass energy $\sqrt{s_{e p}} \simeq$ $1.29 \mathrm{TeV}$ of the $\mathrm{LHeC}$ for the electron beam energy of $60 \mathrm{GeV}$ and proton beam energy of $7 \mathrm{TeV}$, respectively. In order to enrich the statistics, we take into account both single top and single anti-top production processes.

The signal cross sections for the process $e^{-} p \rightarrow\left(e^{-} t+\right.$ $\left.e^{-\bar{t}}\right)+X$ for different values of couplings $\lambda_{u}$ and $\lambda_{c}$ at the LHeC are given in Table 1 . In order to estimate the sensitivity to FCNC coupling parameters in the range, we plot contours corresponding to different values of signal cross sections as shown in Figure 2. A realistic and detailed analysis of the signal and background for one electron, two jets, and one $b$ tagged jet in the final state is given in the following section including a fast detector simulation.

\section{Analysis}

In this section, we study the sensitivity to anomalous top FCNC couplings through the signal and background process $e^{-} p \rightarrow e^{-} W^{ \pm} q+X$ at LHeC. This process includes the off-shell single top quark production and interfering background with the signal. We calculate the cross section for this process to normalize the distributions from the signal and background events. The cross section values depending on the FCNC 

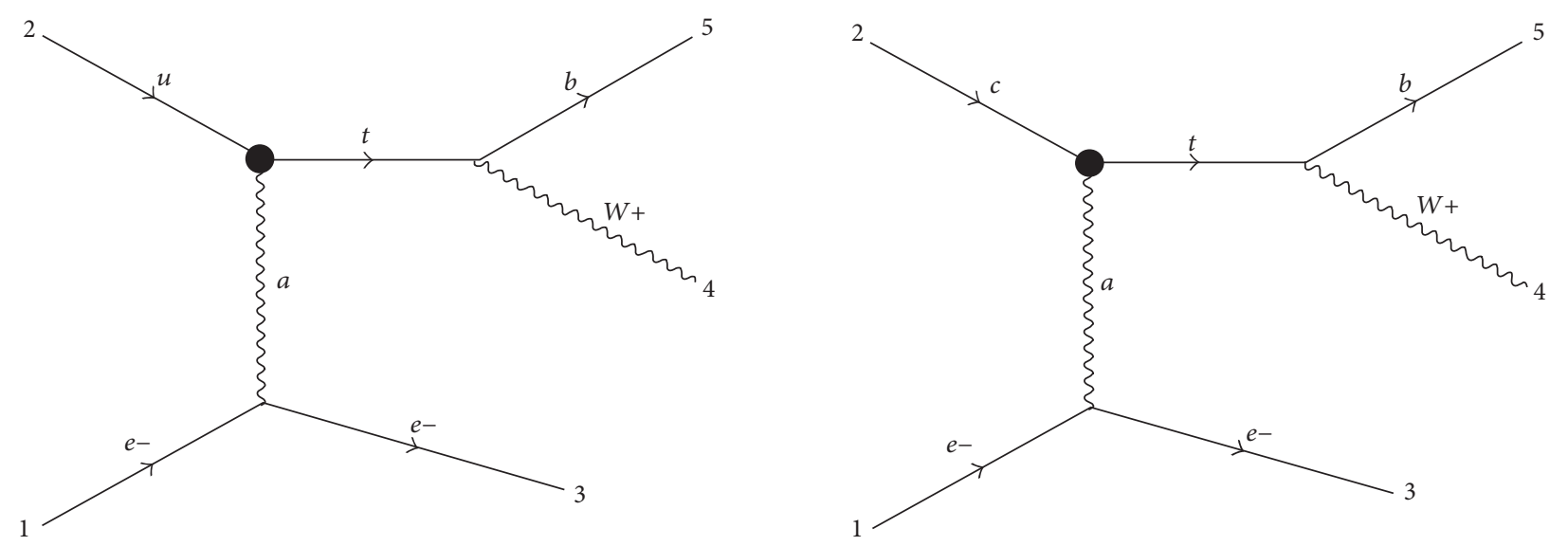

FIGURE 1: Leading order diagrams for single top quark production via flavour changing neutral currents and its subsequent decay. The similar diagrams for single anti-top production can be obtained by exchanging $u \leftrightarrow \bar{u}$ and $c \leftrightarrow \bar{c}$ and its corresponding subsequent decay.

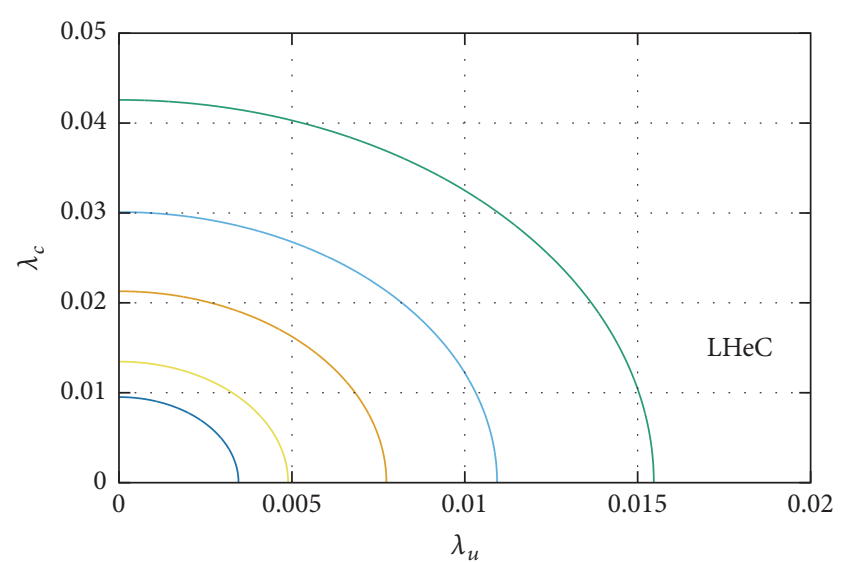

$\sigma(\mathrm{fb})$

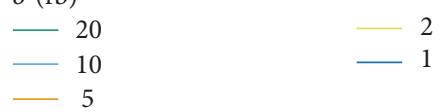

FIGURE 2: Contour plot for plane of FCNC couplings $\lambda_{u}$ and $\lambda_{c}$ depending on signal cross sections at LHeC.

couplings are shown in Table 2 with the generator level preselection cuts $\left(n_{e} \geq 1\right.$ and $\left.n_{j} \geq 3\right)$. We generate signal and background (interfering with signal) events by using MadGraph5_aMC@NLO [19] with the effective Lagrangian implemented through FeynRules [21] model including the CKM matrix elements. Parton showering and fast detector simulations are subsequently performed with Pythia 6 [22] and Delphes 3 [23], respectively. For the analysis of signal and background events, following the generator based preselection cuts, we also apply selection cuts as shown in Table 3 .

Concerning our process $e^{-} p \rightarrow e^{-} W^{ \pm} q+X$, with the hadronic decay mode of the $W$ boson we will have at least three jets and one electron in the final state. We take into account $e W q(B 1)$ and $e Z q(B 2)$ backgrounds which have the same final state after $W$ and $Z$ decays hadronically, as that of the signal process. Here, multijet QCD backgrounds are not included in the analysis. The transverse momentum and
TABLE 2: The cross section (in pb) for the process $e^{-} p \rightarrow e^{-} W^{ \pm} q+X$ depending on the FCNC couplings $\lambda_{u}$ and $\lambda_{c}$. The values correspond to the case that only one of the couplings is allowed to change at a time.

\begin{tabular}{lccccc}
\hline$\lambda_{u}$ or $\lambda_{c} \rightarrow$ & 0.05 & 0.03 & 0.02 & 0.01 & 0 \\
\hline$\lambda_{c}=0$ & 2.493 & 2.368 & 2.329 & 2.307 & 2.298 \\
$\lambda_{u}=0$ & 2.324 & 2.308 & 2.303 & 2.299 & 2.298 \\
$\lambda_{u}=\lambda_{c}$ & 2.519 & 2.378 & 2.333 & 2.307 & 2.298 \\
\hline
\end{tabular}

TABLE 3: The preselection and a set of cuts for the analysis of signal and background events at $\mathrm{LHeC}$.

\begin{tabular}{cc}
\hline Cut-0 & Preselection: $n_{e} \geq 1$ and $n_{\text {jets }} \geq 3$ \\
Cut-1 & $b$-tag: one $b$-tagged jet $\left(j_{b}\right)$ \\
Cut-2 & Transverse momentum: $p_{T}\left(j_{2}, j_{3}\right)>30 \mathrm{GeV}$ and \\
& $p_{T}\left(j_{b}\right)>40 \mathrm{GeV}$ and $p_{T}(e)>20 \mathrm{GeV}$ \\
Cut-3 & Pseudo-rapidity: $-4<\eta\left(j_{b}, j_{2}, j_{3}\right)<0$ and $|\eta(e)|<2.5$ \\
Cut-4 & $W$ boson mass: $50<M_{\mathrm{inv}}^{\mathrm{rec}}\left(j_{2}, j_{3}\right)<100 \mathrm{GeV}$ \\
Cut-5 & Top quark mass: $130<M_{\mathrm{inv}}^{\mathrm{rec}}\left(j_{b}+j_{2}+j_{3}\right)<190 \mathrm{GeV}$ \\
\hline
\end{tabular}

pseudo-rapidity distributions of the leading jet and second and third jets are presented in Figure 3 for the signal and interfering background together, and in Figure 4 only the backgrounds $B 1$ and $B 2$. The distribution has similar shape for signal and considers backgrounds. In order to select signal events, each event is required to have one electron, two jets, and one $b$-jet with the highest $p_{T}$. The number of selected objects in the signal events can help to suppress the background events effectively. The kinematical distributions $\left(p_{T}(e)\right.$ and $\left.\eta(e)\right)$ for the electron in the event are shown in Figure 5 for $S+B 1$ and in Figure 6 for only $B 1$ and B2. One of the specific signatures of the signal is the presence of an electron with high transverse momentum $\left(p_{T}(e)>20 \mathrm{GeV}\right)$ and central pseudo-rapidity $(|\eta(e)|<2.5)$. The leading jet with $p_{T}(j)>40 \mathrm{GeV}$ and other two jets with $p_{T}(j)>$ $30 \mathrm{GeV}$ are considered in our analysis, as the cuts flow shown in Table 3. Since the $\mathrm{LHeC}$ has an energy asymmetry, the jets from the process mainly peaked in the backward region 

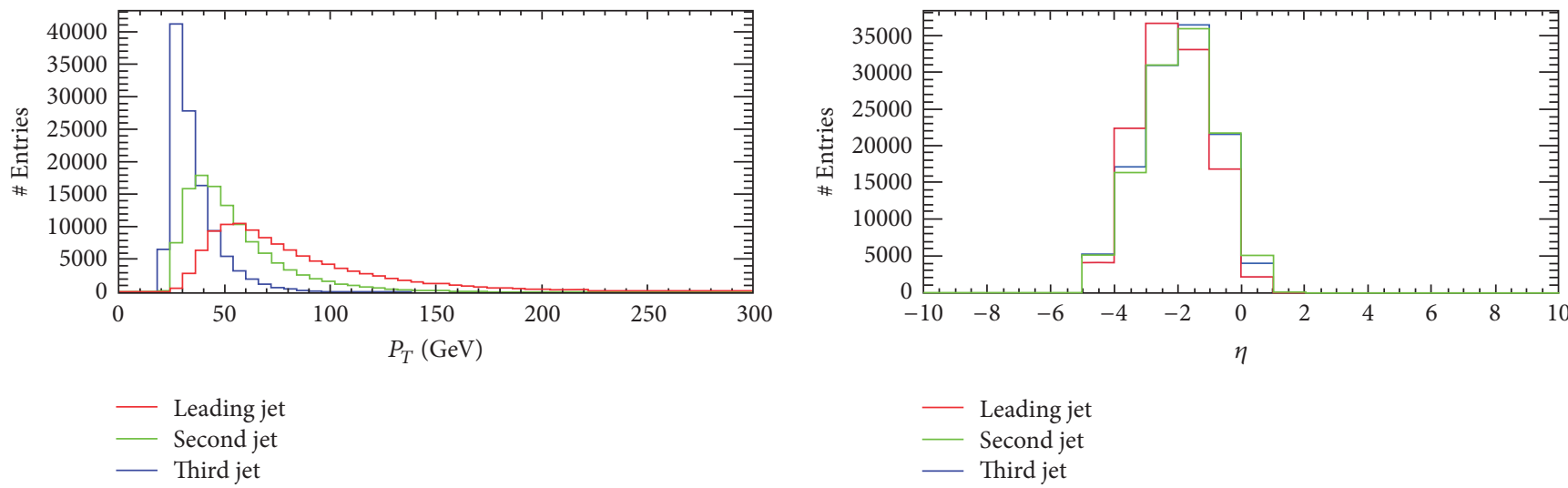

FIGURE 3: Transverse momentum and pseudo-rapidity distributions of three jets from the process $e^{-} p \rightarrow e^{-} W^{ \pm} q+X$ which includes both the interfering background and signal for $\lambda_{u}=\lambda_{c}=0.05$ at the LHeC.
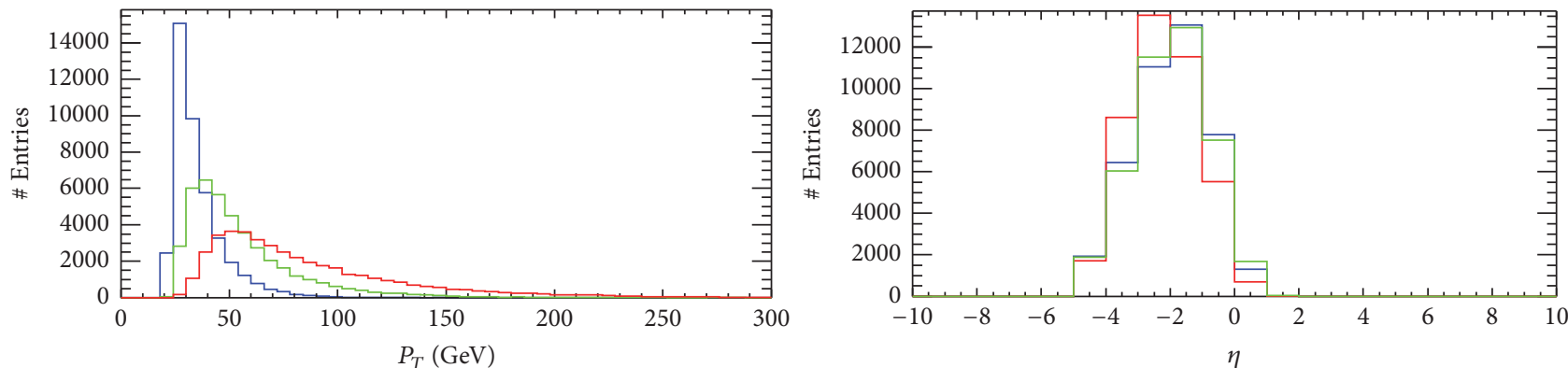

(a)
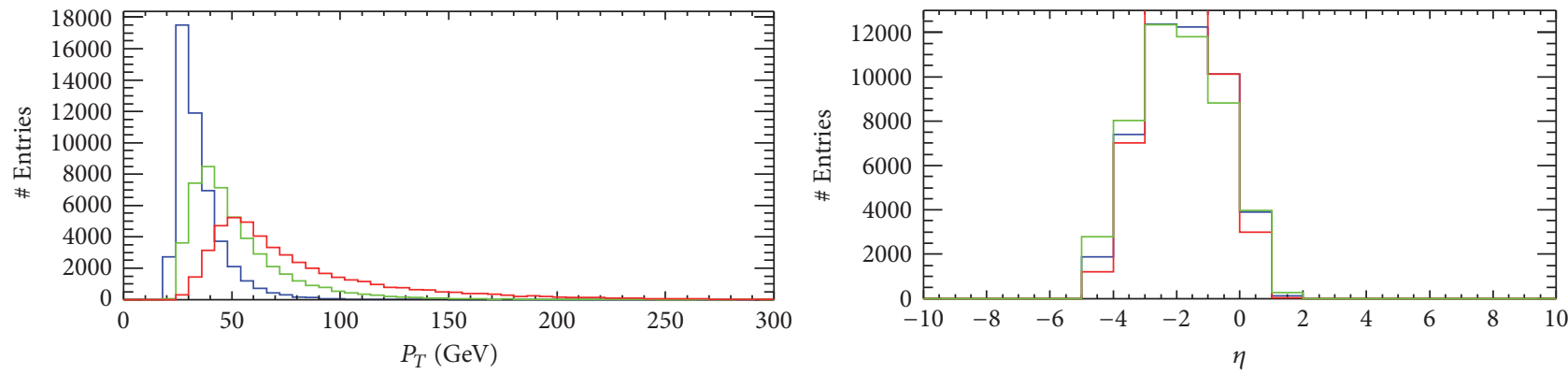

(b)

FIgURE 4: Transverse momentum and pseudo-rapidity distributions of three jets from the process $e^{-} p \rightarrow e^{-} W^{ \pm} q+X$ for background (B1) (a) and the process $e^{-} p \rightarrow e^{-} Z q+X$ for background (B2) (b) at the LHeC.
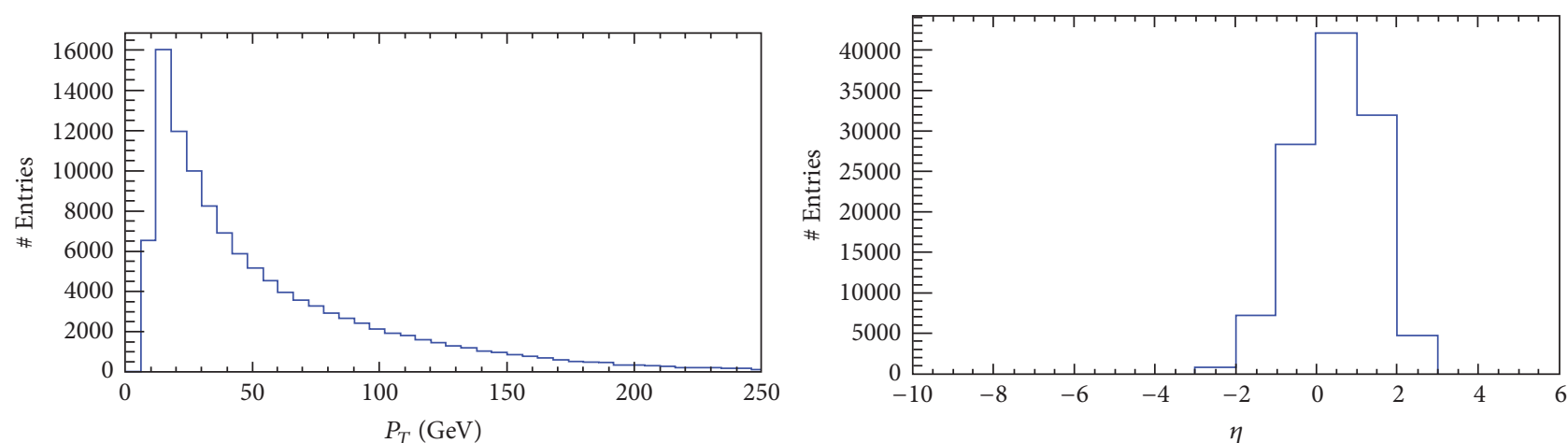

FiguRE 5: Transverse momentum and pseudo-rapidity distribution of electron from the process $e^{-} p \rightarrow e^{-} W^{ \pm} q+X$ which includes both the background and signal for $\lambda_{u}=\lambda_{c}=0.05$ at the LHeC. 


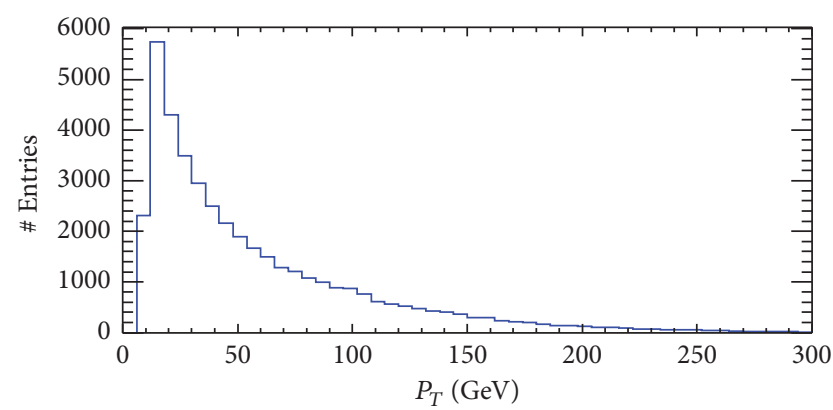

(a)

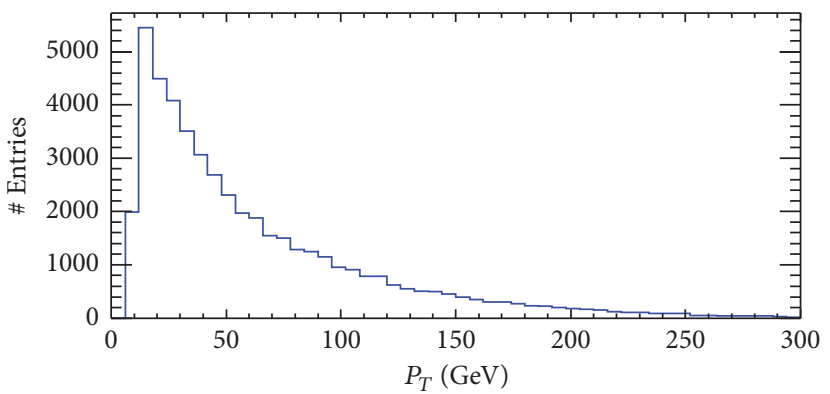

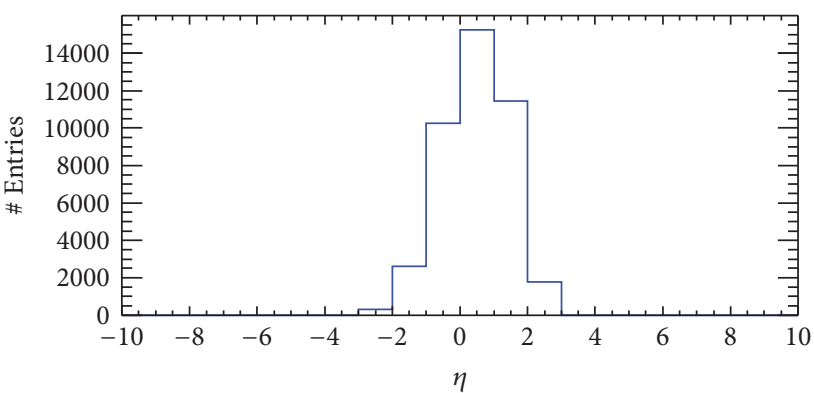

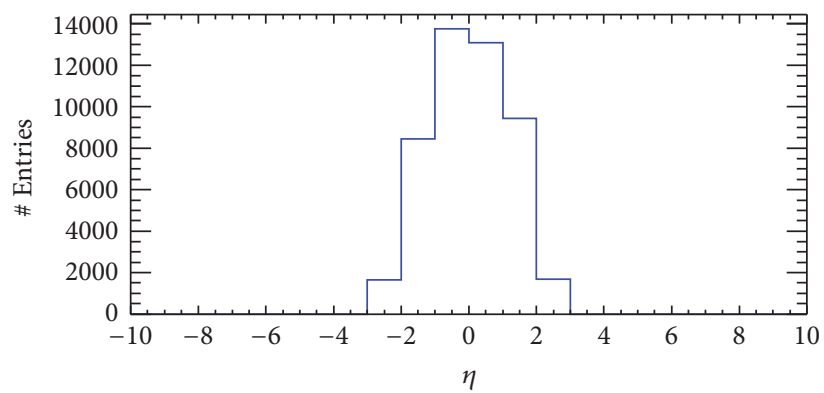

(b)

FIgURE 6: Transverse momentum and pseudo-rapidity distribution of electron from the process $e^{-} p \rightarrow e^{-} W^{ \pm} q+X$ for background (B1) (a) and the process $e^{-} p \rightarrow e^{-} Z q+X$ for background (B2) (b) at the LHeC.

according to the ep collisions; therefore the pseudo-rapidity range for jets is taken to be $-4<\eta(j)<0$ for the analysis.

In the fast simulation, the detector card includes $b$ tagging efficiency to be $70 \%$, with mistagging efficiencies for $c$-jets and light jets of $10 \%$ and $0.3 \%$, respectively, in the considered $\left(p_{T}, \eta\right)$ range of the jets. These are consistent with the typical values used by the LHC experiments [24]. The top quark mass is reconstructed from three jets (with one $b$ tagged jet) after selection cuts. At each cut step we calculate the cut efficiencies as shown in Figure 7 for different FCNC coupling parameter. This figure shows that the cut efficiency changes from $6 \%$ to $1 \%$ for interfering backgrounds $\mathrm{eWq}$ $(B 1)$ and $e Z q(B 2)$ with cut steps from Cut-1 to Cut-5 when the FCNC coupling $(\lambda)$ is set to zero. For the signal the cut efficiency depends on the value of the couplings, for example, it decreases from $10 \%$ to $2.5 \%$ for the couplings $\lambda_{u}=\lambda_{c}=$ 0.05 .

The number of events for signal and backgrounds after Cut-5 at LHeC with an integrated luminosity of $L_{\text {int }}=$ $100 \mathrm{fb}^{-1}$ are given in Table 4. The respective number of events after each cut can be obtained from Table 4 with a relative factor.

We plot the invariant mass of top quark (reconstructed from three jets, one is $b$-tagged) as shown in Figure 8 . The Cut- 4 for $W$ boson mass window reduces $e^{-} Z q$ background (B2) effectively, while keeping the signal less affected. The invariant mass $\left(M_{j j j}\right)$ distribution after Cut-4 and ratio $(S+$ $B 1+B 2) /(B 1+B 2)$ plot for equal coupling scenario $\left(\lambda_{u}=\right.$ $\left.\lambda_{c}=0.05\right)$ are presented in Figure 8, where the main contribution comes from the $B 1$ background.

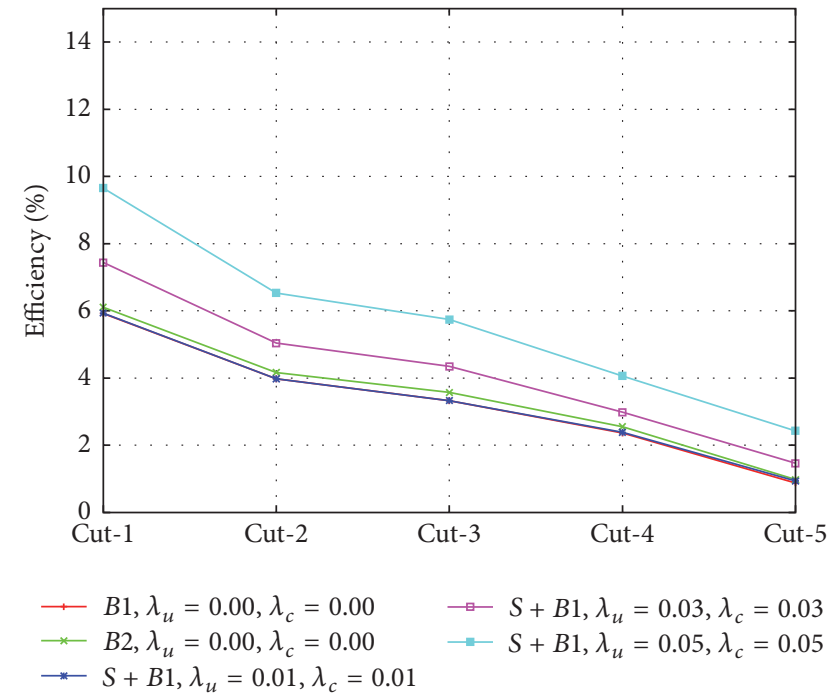

FIGURE 7: Efficiency plot for the cuts applied at each step for the analysis of signal $(S)+$ background $(B 1)$ and background (B2) events. The cut efficiencies are calculated with respect to the preselection cuts for each coupling value.

The statistical significance (SS) is calculated at the final stage of the cuts using the signal $(S)$ and total background $(B)$ events

$$
S S=\sqrt{2\left[(S+B) \ln \left(1+\frac{S}{B}\right)-S\right]} .
$$




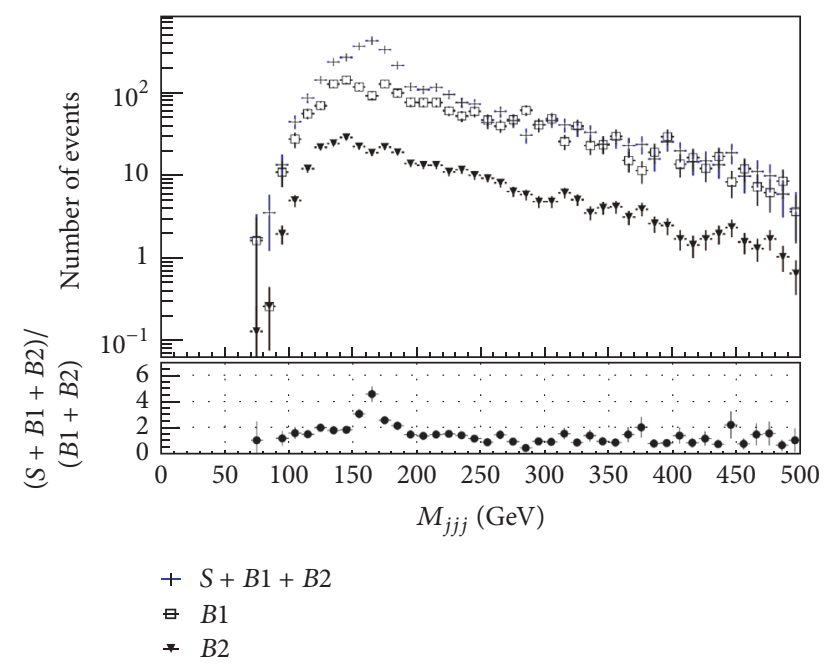

FIGURE 8: Invariant mass distributions of three jets (one of the jets is required as $b$-jet) for the signal + background $(S+B 1+B 2)$ and backgrounds $(B 1, B 2)$. The ratio plot presents the signal (for equal coupling scenario $\lambda=0.05$ ) strength which peaks at the top mass.

TABLE 4: The number of events for background $B 1$ (B2) and signal with FCNC couplings $\lambda_{u}$ and $\lambda_{c}$ at LHeC with $L_{\text {int }}=100 \mathrm{fb}^{-1}$.

\begin{tabular}{lcccc}
\hline & $\lambda_{c}=0$ & $\lambda_{c}=0.01$ & $\lambda_{c}=0.03$ & $\lambda_{c}=0.05$ \\
\hline$\lambda_{u}=0$ & $584(149)$ & 592 & 609 & 640 \\
$\lambda_{u}=0.01$ & 617 & 621 & 692 & 763 \\
$\lambda_{u}=0.03$ & 943 & 969 & 1003 & 1209 \\
$\lambda_{u}=0.05$ & 1502 & 1744 & 1758 & 1792 \\
\hline
\end{tabular}

For different coupling scenario $\left(\lambda_{u} \neq \lambda_{c}\right)$, we calculate the significance and search for the sensitivity to the FCNC couplings $\lambda_{u}$ and $\lambda_{c}$. The SS reach for FCNC tq $\gamma$ couplings is presented in Figures 9 and 10 depending on the integrated luminosity ranging from $1 \mathrm{fb}^{-1}$ to $1 \mathrm{ab}^{-1}$ at the LHeC. It includes the contribution from the main background on the predicted results. The signal significance corresponding to $2 \sigma, 3 \sigma$, and $5 \sigma$ lines is also shown. In Figure 11 we also present a plot showing the $3 \sigma$ and $5 \sigma$ contours in the $\lambda_{u}-\lambda_{c}$ plane at $\mathrm{LHeC}$ for an integrated luminosity of $500 \mathrm{fb}^{-1}$. In this study, we have calculated the cross section with the theoretical uncertainties for the PDF scale variation which is in the range $\pm 2.5 \%$. The LHeC collider would be clear environment and the systematics would be less than that of the LHC. For the sake of clarity, only uncertainties from statistics are taken into account, while the uncertainties from systematics are ignored in the present discussions.

In the literature, there are alternative uses of effective coupling constants appearing in the effective Lagrangian. It is also useful to express our results in terms of branching ratios which can be comparable with the results of other studies. In order to translate the bounds, we define the branching ratio as

$$
\begin{aligned}
& \operatorname{BR}(t \longrightarrow q \gamma) \\
& \quad=\frac{\Gamma(t \longrightarrow q \gamma)}{\Gamma\left(t \longrightarrow q^{\prime} W^{+}\right)+\Gamma(t \longrightarrow u \gamma)+\Gamma(t \longrightarrow c \gamma)} .
\end{aligned}
$$

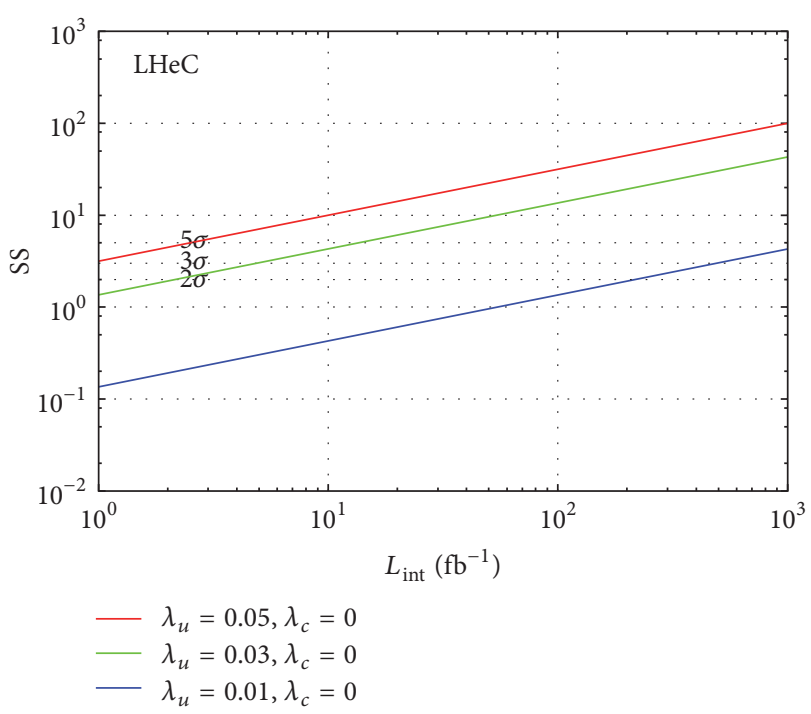

FIGURE 9: Estimated statistical significance (SS) reach of flavour changing neutral current tu $\gamma$ coupling $\left(\lambda_{u}\right)$ depending on the integrated luminosity ranging from $1 \mathrm{fb}^{-1}$ to $1 \mathrm{ab}^{-1}$ at the LHeC. It includes the contribution from the main background $(B 1)$ on the predicted results. The signal significance corresponding to $2 \sigma, 3 \sigma$, and $5 \sigma$ lines is also shown.

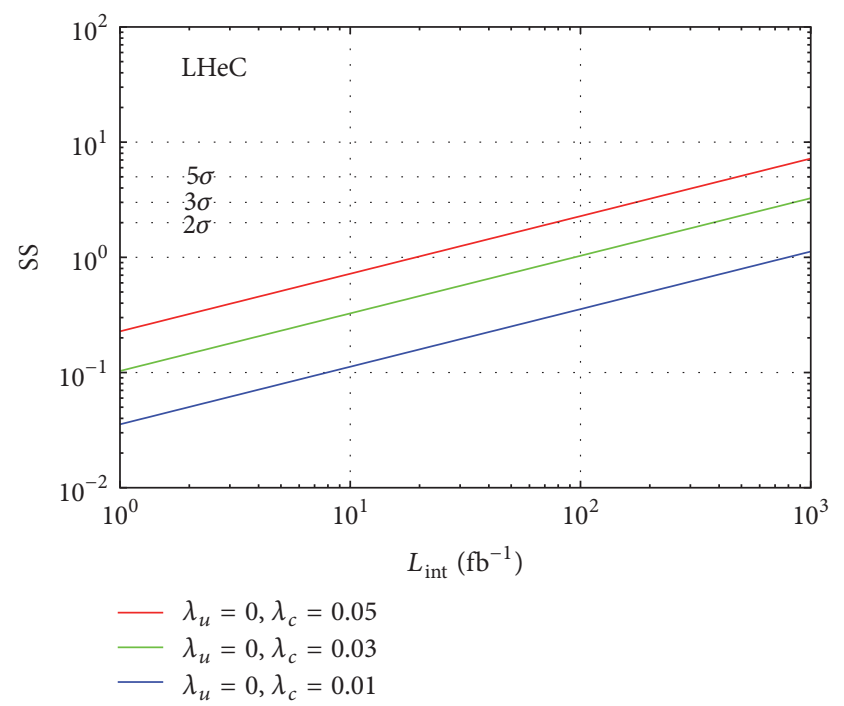

FIGURE 10: Estimated statistical significance (SS) reach of flavour changing neutral current $t c \gamma$ coupling $\left(\lambda_{c}\right)$ depending on the integrated luminosity ranging from $1 \mathrm{fb}^{-1}$ to $1 \mathrm{ab}^{-1}$ at the LHeC. It includes the contribution from the main background (B1) on the predicted results. The signal significance corresponding to $2 \sigma, 3 \sigma$, and $5 \sigma$ lines is also shown.

Since the $V_{t b}$ element of CKM matrix is much larger than $V_{t s}$ and $V_{t d}$, the main contribution to $\Gamma\left(t \rightarrow q^{\prime} W^{+}\right)$comes from the decay $t \rightarrow b W$ for a value of about $\Gamma(t \rightarrow b W)=1.41 \mathrm{GeV}$ [25]. We calculate the partial widths for the FCNC decay channels $t \rightarrow q \gamma$ as $\Gamma(t \rightarrow q \gamma)=(1 / 8) \alpha_{e} \lambda_{q}^{2} m_{t}$.

In this study, we find $3 \sigma$ significance results to reach upper limits $\lambda_{u}=0.012$ and $\lambda_{c}=0.032$ with an integrated 


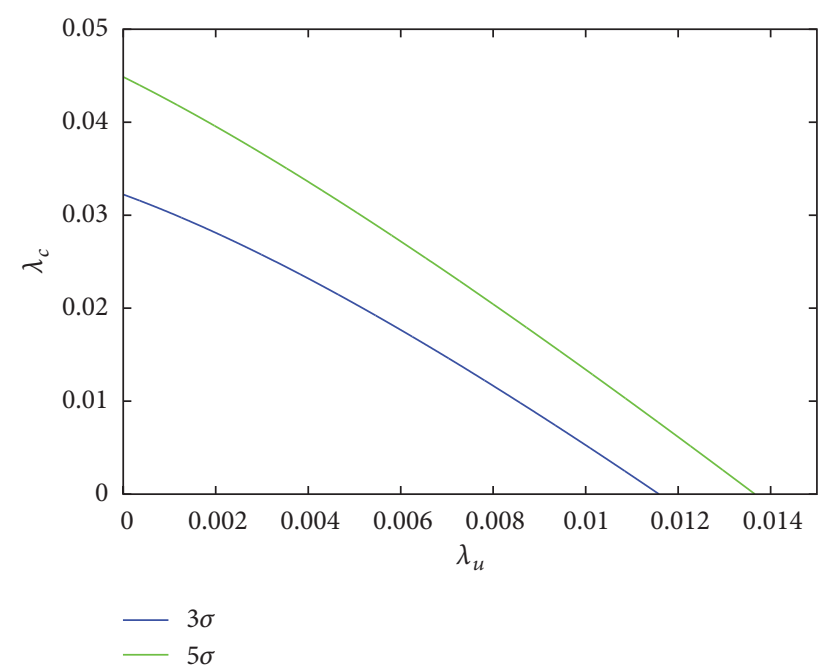

FIGURE 11: The contour plot for the couplings $\lambda_{u}$ and $\lambda_{c}$ at LHeC for an integrated luminosity of $500 \mathrm{fb}^{-1}$.

luminosity of $500 \mathrm{fb}^{-1}$ at $\mathrm{LHeC}$. These limits on the couplings can also be translated to the upper bounds on branching ratios $\mathrm{BR}(t \rightarrow u \gamma)<1.62 \times 10^{-5}$ and $\mathrm{BR}(t \rightarrow c \gamma)<$ $1.15 \times 10^{-4}$, respectively. Previous experimental constraints on the branching fractions of $t \rightarrow q \gamma$ (with $q$ representing an up or charm quark) from the $e p$ collider HERA are $0.29 \%$ from ZEUS [26] and $0.64 \%$ from H1 [27] at the $95 \%$ confidence level (CL). At LHeC energy region the $c$-quark contribution to the process becomes comparable with the $u$-quark contribution; hence the sensitivity to $\lambda_{c}$ will be enhanced comparing to HERA results. We can also compare our results with the recent results from the CMS experiment with an integrated luminosity of $19.8 \mathrm{fb}^{-1}$ which place an upper bound on top quark FCNC branching ratios $\mathrm{BR}(t \rightarrow$ $u \gamma)<1.61 \times 10^{-4}$ and $\mathrm{BR}(t \rightarrow c \gamma)<1.82 \times 10^{-3}$ at $95 \%$ confidence level [6]. Our results show that an order of magnitude improvement can be achieved for the top quark FCNC branching.

\section{Conclusion}

The $\mathrm{LHeC}$ projected as an ep collider, especially due to its respective clean environment when compared to high energy hadron colliders and the electron beam polarization possibility, will provide important results for some certain processes. In this study, we focused on the physics potential of the $\mathrm{LHeC}$ promoting its complementary and competitive role. We have analyzed the process $e^{-} p \rightarrow e^{-} W^{ \pm} q+X$ with the signature including one isolated electron and one $b$-jet together with two jets in the final state. The signal for this process includes the top quark flavour changing neutral current couplings (tq $)$ through photon exchanges in electron-proton collisions. We set upper limits on the top quark FCNC couplings from the analysis of signal and background including detector effects through the fast simulation. The expected limits on tq $\gamma$ couplings at HL-LHC have already been reported in [28]; the branching ratios for $t \rightarrow q \gamma$ are $8 \times 10^{-5}$ and $2.5 \times 10^{-5}$ for $L_{\text {int }}=300 \mathrm{fb}^{-1}$ and $3000 \mathrm{fb}^{-1}$, respectively. The $\mathrm{LHeC}$ with the high luminosity of $1 \mathrm{ab}^{-1}$ has the potential of probing the top FCNC couplings $\left(\lambda_{u}, \lambda_{c}\right)$, which can be comparable or even better when compared to the bounds from the HL-LHC.

\section{Conflicts of Interest}

The authors declare that there are no conflicts of interest regarding the publication of this paper.

\section{Acknowledgments}

The authors acknowledge illuminating discussions within the LHeC Higgs and Top group. I. Turk Cakir would like to thank CERN for the hospitality, where a part of this work has been completed.

\section{References}

[1] S. L. Glashow, J. Iliopoulos, and L. Maiani, "Weak interactions with lepton-hadron symmetry," Physical Review D, vol. 2, no. 7, pp. 1285-1292, 1970 .

[2] J. A. Aguilar-Saavedra, "Top flavour-changing neutral interactions: theoretical expectations and experimental detection," Acta Physica Polonica B, vol. 35, article 2695, 2004.

[3] G. Couture, M. Frank, and H. König, "Supersymmetric QCD flavor-changing top quark decay," Physical Review D, vol. 56, no. 7, pp. 4213-4218, 1997.

[4] G. Lu, F. Yin, X. Wang, and L. Wan, "Rare top quark decays," Physical Review D, vol. 68, no. 1, 2003.

[5] T. Aaltonen, "Erratum: measurement of the inclusive leptonic asymmetry in top-quark pairs that decay to two charged leptons at CDF," Physical Review Letters, vol. 117, article 2525, no. 19, 2016.

[6] CMS Collaboration, "Search for anomalous single top quark production in association with a photon in pp collisions at $\mathrm{s} \sqrt{ }=8$ TeV," Journal of High Energy Physics, 2014.

[7] J. de Favereau de Jeneret and S. Ovyn, "Single top quark photoproduction at the LHC," Nuclear Physics B - Proceedings Supplements, vol. 179-180, no. C, pp. 277-284, 2008.

[8] "Probe anomalous tq $\gamma$ couplings through single top photoproduction at the LHC", Nuclear Physics B, vol. 886, pp. 691-711, 2014.

[9] K. Bamba and S. D. Odintsov, "Inflation in a viscous fluid model," The European Physical Journal C, vol. 76, p. 18, 2016.

[10] K. Kanazawa, Y. Koike, A. Metz, and D. Pitonyak, “Transverse single-spin asymmetries in $p^{\uparrow} p \rightarrow \gamma X$ from quark-gluonquark correlations in the proton," Physical Review D, vol. 91, Article ID 014013, 2015.

[11] H. Denizli, A. Senol, A. Yilmaz, I. T. Cakir, H. Karadeniz, and O. Cakir, "Top quark FCNC couplings at future circular hadron electron colliders," Physical Review D, vol. 96, no. 1, 2017.

[12] LHeC Study Group., J. L Abelleira Fernandez et al., Journal of Physics G: Nuclear and Particle Physics, vol. 39, Article ID 075001, 2012.

[13] M. Klein, "Deep inelastic scattering at the energy frontier," Annalen der Physik, vol. 528, no. 1-2, pp. 138-144, 2016. 
[14] J. L. Abelleira Fernandez, C. Adolphsen, and A. N. Akay, "A large hadron electron collider at CERN. Report on the physics and design concepts for machine and detector," Journal of Physics $G$, vol. 39, Article ID 075001, 2012.

[15] M. Kumar, X. Ruan, R. Islam et al., "Probing anomalous couplings using di-Higgs production in electron-proton collisions," Physics Letters, Section B: Nuclear, Elementary Particle and HighEnergy Physics, vol. 764, pp. 247-253, 2017.

[16] X. Yuan, Y. Hao, and Y. Yang, " $\bar{B} \rightarrow X_{s} \gamma$ constraints on the top quark anomalous $t \rightarrow c \gamma$ coupling," Physical Review D, vol. 83, no. 1, 2011.

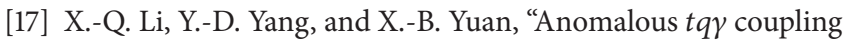
effects in exclusive radiative B-meson decays," Journal of High Energy Physics, vol. 08, p. 075, 2011.

[18] Y. D. Yang and X. B. Yuan, "Anomalous top-quark coupling effects in rare B and K decays," Chinese Science Bulletin, vol. 59, p. 3760, 2014.

[19] J. Alwall, M. Herquet, F. Maltoni, O. Mattelaer, and T. Stelzer, "MadGraph 5: going beyond," Journal of High Energy Physics, vol. 128, 2011.

[20] R. D. Ball et al., "NNPDF Collaboration," Nuclear Physics B, vol. 867, no. 2, pp. 244-289, 2013.

[21] A. Alloul, N. D. Christensen, C. Degrande, C. Duhr, and B. Fuks, "FeynRules 2.0 - A complete toolbox for tree-level phenomenology," Computer Physics Communications, vol. 185, no. 8, pp. 2250-2300, 2014.

[22] T. Sjöstrand, S. Mrenna, and P. Skands, "PYTHIA 6.4 physics and manual," Journal of High Energy Physics, vol. 5, article 026, 2006.

[23] J. de Favereau, C. Delaere, P. Demin et al., "DELPHES 3: a modular framework for fast simulation of a generic collider experiment," Journal of High Energy Physics, vol. 2014, article 57, 2014.

[24] ATLAS Collaboration., "Expected performance of the ATLAS b-tagging algorithms in Run-2," ATL-PHYS-PUB-2015-022, 2015.

[25] C. Patriagnani et al., "Particle Data Group," Chinese Physics C, vol. 40, Article ID 100001, 2016.

[26] ZEUS Collaboration., H. Abramowicz, I. Abt et al., "Search for single-top production in ep collisions at HERA," Physics Letters $B$, vol. 708, no. 1-2, pp. 27-36, 2012.

[27] H1 Collaboration., F. D. Aaron, M. Aldaya Martin et al., "Search for single top quark production at HERA," Physics Letters B, vol. 678, no. 5, pp. 450-458, 2009.

[28] “ATLAS Collaboration," https://arxiv.org/abs/1307.7292. 

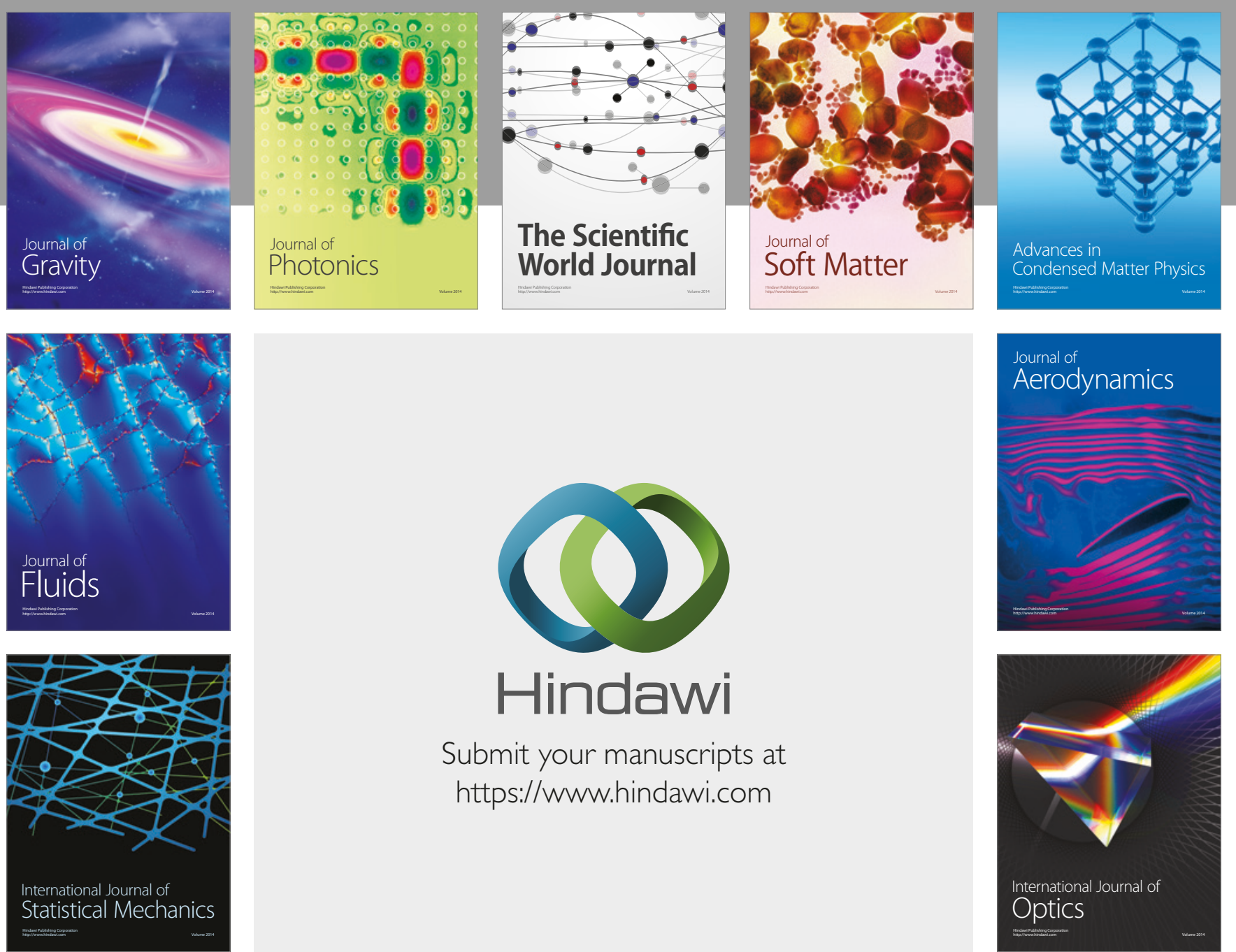

Submit your manuscripts at

https://www.hindawi.com
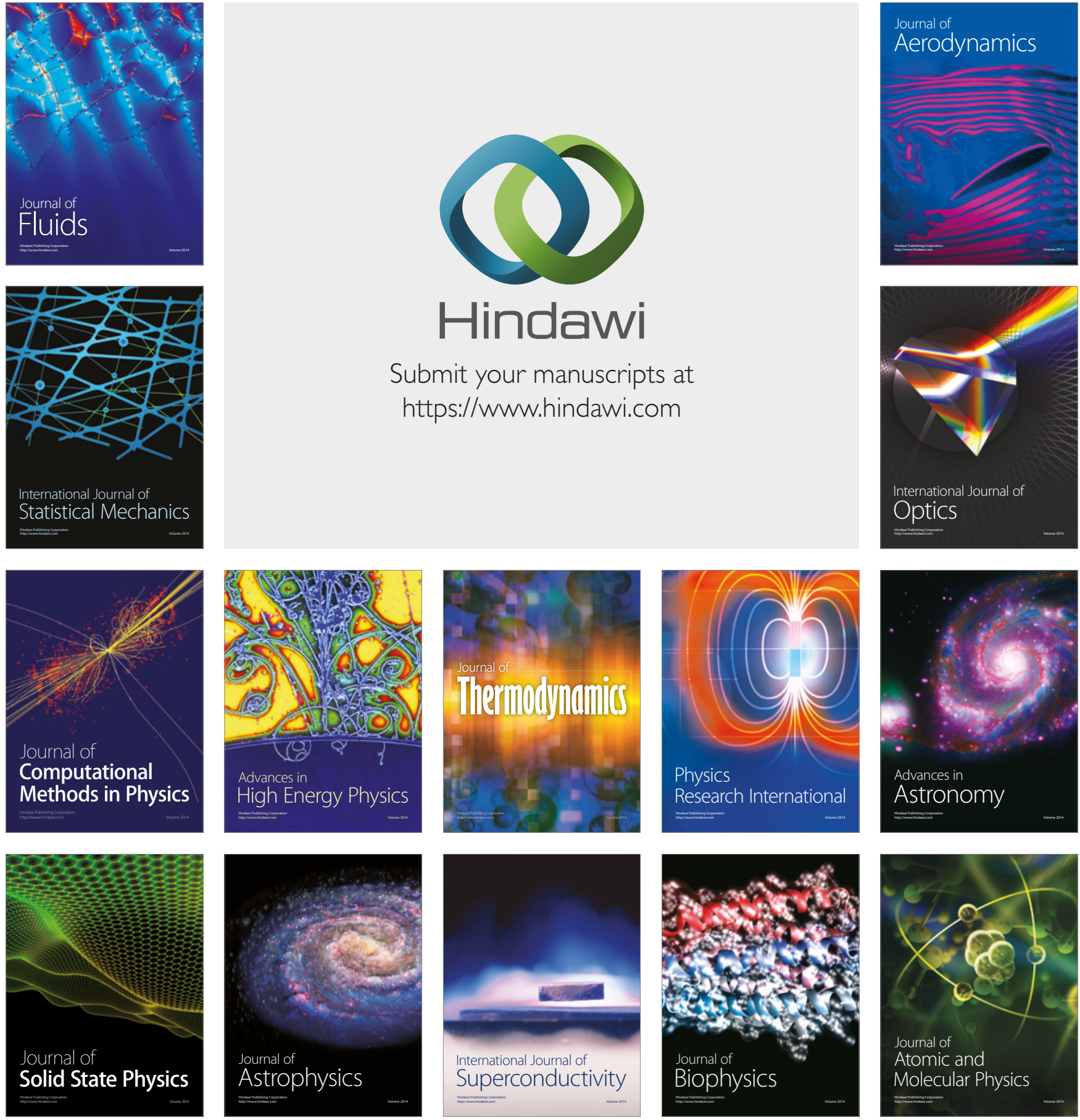